\title{
Exploring the limits of granular hydrodynamics: A horizontal array of inelastic particles
}

\author{
Peter Eshuis, ${ }^{1}$ Ko van der Weele, ${ }^{2}$ Enrico Calzavarini, ${ }^{1,3}$ Detlef Lohse, ${ }^{1}$ and Devaraj van der Meer ${ }^{1}$ \\ ${ }^{1}$ Physics of Fluids Group and J. M. Burgers Center for Fluid Dynamics, University of Twente, \\ P.O. Box 217, 7500 AE Enschede, The Netherlands \\ ${ }^{2}$ Department of Mathematics, Division of Applied Analysis, University of Patras, 26500 Patras, Greece \\ ${ }^{3}$ Laboratoire de Physique, Ecole Normale Supérieure de Lyon, CNRS UMR 5672, 46 allée d'Italie, 69007 Lyon, France
}

(Received 24 February 2009; published 2 July 2009)

\begin{abstract}
The limits of granular hydrodynamics are explored in the context of the one-dimensional granular system introduced by Du, Li, and Kadanoff [Phys. Rev. Lett. 74, 1268 (1995)]. The density profile of the characteristic steady state, in which a single particle commutes between the driving wall and a dense cluster, is well captured by a hydrodynamic description provided that the finite size of the particles is incorporated. The temperature, however, is not well described: since all energy exchange is located at the border of the cluster, it is precisely for this quantity that the continuum approach breaks down.
\end{abstract}

DOI: 10.1103/PhysRevE.80.011302

PACS number(s): 45.70.-n, 05.65.+b, 05.20.Dd

\section{INTRODUCTION}

One of the central themes in the field of granular matter is the question to what extent the rich variety of experimental phenomena can be captured by hydrodynamic continuum theory. Such a theory can hardly be expected to cover all observed effects [1], the main obstacle being the lack of separation of scales: the average distance between neighboring granular particles is not negligible compared to the system size. This is a serious limitation to any continuum theory, especially for the small-scale phenomena. For largescale collective effects, however, hydrodynamic modeling is a natural approach $[2,3]$ and has been successfully applied to a large number of phenomena ranging from cluster formation in various granular gases [4-6] to convection rolls in a vibrated granular bed [7] or in chute flow down an inclined plane [8], the fluidlike impact of a steel ball on sand [9], and the granular Leidenfrost effect [10].

A very illustrative example in this context was introduced in 1995 by $\mathrm{Du}, \mathrm{Li}$, and Kadanoff [11]. It consists of $N$ inelastically colliding, sizeless particles confined to a horizontal tube [Fig. 1(a)] driven at the left wall: a random velocity is given to the leftmost particle every time it hits this wall. The right wall is insulating, i.e., the collisions of the rightmost particle with this wall are fully elastic.

Starting out from a homogeneous distribution, the particles are seen to cluster at the right wall [Fig. 1(b)]. All particles get caught in the cluster, except the leftmost particle, which keeps traveling back and forth between the hot wall and the cluster $[11,12]$. Clearly, there is no equipartition of energy: A dilute region consisting of one fast particle coexists with a dense region of slow particles. A typical timeaveraged density and temperature distribution are shown in Figs. 1(c) and 1(d). Du et al. [11] demonstrated that the "simplest hydrodynamic approach," treating the system as an ideal gas of sizeless particles with energy dissipation (from the particle collisions), fails to correctly describe this state.

What is the reason for this failure? As it turns out, the crucial point is that the individual left particle has no way of establishing a continuous energy exchange along its path. It therefore does not form a gas in the hydrodynamic sense, but rather a Knudsen gas, which is by definition so dilute that the particle collisions within the gas can be ignored in comparison with the collisions with the boundaries. In the onedimensional system of $\mathrm{Du}$ et al. [11] the dilute region contains only a single particle, so not even one collision occurs in this Knudsen gas region.

In this paper we now include the finite size of the particles in both model and simulation. We show that (when the excluded volume is properly accounted for) hydrodynamics is able to capture the density throughout the system, but not the energy profile. Therefore this one-dimensional system not only indicates where the continuum theory breaks down but also the reason why.

It is known that one-dimensional granular systems behave qualitatively different from two- and three-dimensional sys-

(a)

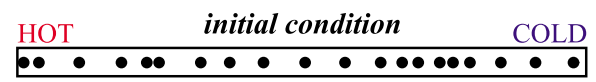

(b)

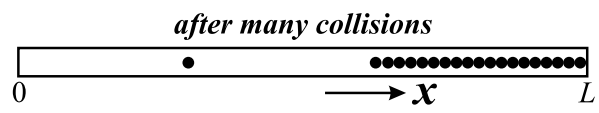

(c)
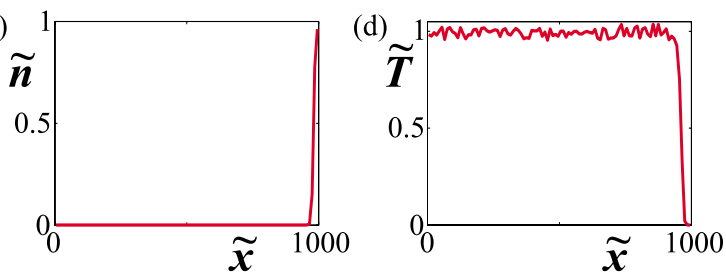

FIG. 1. (Color online) (a) Initial condition of a molecular dynamics simulation with $N=20$ identical particles, diameter $d$, randomly distributed over the tube length $L$. Every time the leftmost particle hits the left wall it is given a random velocity. The collisions of the rightmost particle with the right wall are elastic. (b) After many inelastic collisions, a cluster of slow particles is kept close to the right wall by one relatively fast particle commuting between the hot wall and the cluster. (c) Time-averaged number density $\tilde{n}(\widetilde{x})$ of the steady state and (d) the corresponding granular temperature $\widetilde{T}(\widetilde{x})$ for $L=1000 d$. (The tilde above $\widetilde{x}, \widetilde{n}$ and $\widetilde{T}$ indicates that these are dimensionless quantities that will be introduced later.) 


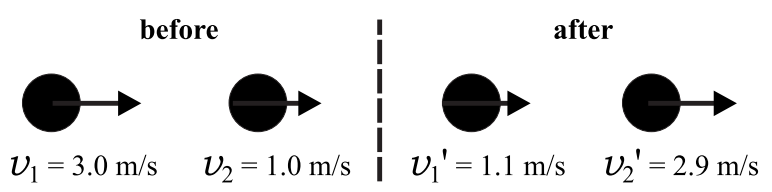

FIG. 2. Example of an inelastic collision of two identical particles. The initial and final velocities are indicated: their sum is constant $\left(v_{1}+v_{2}=v_{1}^{\prime}+v_{2}^{\prime}\right)$, expressing momentum conservation, whereas the difference becomes smaller and defines the restitution coefficient $e=-\left(v_{2}^{\prime}-v_{1}^{\prime}\right) /\left(v_{2}-v_{1}\right)=0.9$.

tems since the latter are in general fairly well-described by granular hydrodynamics $[1,6,10,13,14]$. The physical reason for this difference is that particles cannot get past each other in one dimension. For the system discussed here this enables the one particle on the left to single-handedly control the cluster on the right. In two or more dimensions such a control is only possible (if at all) in a statistical sense, since there particles do pass each other $[6,10,13,14]$. This highlights the significance of this system: it is not just an example in which hydrodynamics fails, but actually marks the border of granular hydrodynamics.

The structure of the paper is as follows: in Sec. II we discuss the particle simulations and results. Subsequently, in Sec. III we turn to the hydrodynamic modeling of the system. The fact that this model takes the finite particle size into account represents the key difference with the approach followed in Du et al. [11]. After that, in Sec. IV we deal with the fact that the diluted phase consists of a single particle and hence forms a Knudsen gas. Section V contains concluding remarks.

\section{MOLECULAR DYNAMICS SIMULATIONS}

We use an event-driven molecular dynamics (MD) code, in which $N$ identical particles [15] of diameter $d$ and unit mass $m$ collide inelastically [16]. The velocities after each collision are related to those before the collision (see Fig. 2) by the following two rules:

$$
\begin{aligned}
& v_{1}^{\prime}=\frac{1}{2}\left[(1-e) v_{1}+(1+e) v_{2}\right], \\
& v_{2}^{\prime}=\frac{1}{2}\left[(1+e) v_{1}+(1-e) v_{2}\right] .
\end{aligned}
$$

They are derived from the conservation of momentum $\left(v_{1}^{\prime}+v_{2}^{\prime}=v_{1}+v_{2}\right)$ together with the definition of $e$, the coefficient of restitution: $v_{1}^{\prime}-v_{2}^{\prime}=-e\left(v_{1}-v_{2}\right)$. If $e=1$ the collisions are fully elastic, but we will consider only the inelastic case $e<1$, in which the particles lose a fraction $\varepsilon=\left(1-e^{2}\right)$ of their kinetic energy in every collision. In order to avoid inelastic collapse (an infinite number of collisions in a finite time $[11,17])$ we choose $N \varepsilon<1$.

The left wall is hot: it drives the leftmost particle by giving it a random velocity from a linearly corrected Gaussian distribution $v_{0} \exp \left(-v_{0}^{2} / 2 T_{0}\right)[1,18]$. The linear prefactor $v_{0}$ corrects for the fact that a small velocity (given to the leftmost particle) resides longer in the system than a large one since it takes longer to travel up and down the whole length of the tube (and thus has a larger influence on the time average); it ensures that the ensemble of all $N$ particles acquires a time-averaged velocity distribution that is purely Gaussian in the elastic case $(e=1)$. In order to minimize the transient time before this distribution establishes itself, we initially put the particles at random positions in the tube and give each of them a random velocity picked from the same (linearly corrected) distribution as we use for the leftmost particle when it hits the hot wall.

The value of $T_{0}$ (the granular temperature of the hot wall) gives the width of the velocity distribution offered to the leftmost particle, i.e., the strength of the driving. The temperature is defined by $\frac{1}{2} k_{B} T=\frac{1}{2} m\left(\left\langle v^{2}\right\rangle-\langle v\rangle^{2}\right)$ with $k_{B}=1$ (the standard choice for granular systems, giving $T$ the dimensions of energy) and unit mass $m$. The right wall is insulating: the collisions of the rightmost particle with this wall are perfectly elastic, so no energy is dissipated here.

Figure 1 shows the result of a typical MD simulation with $N=20$ particles. Steady state profiles for higher values of $N$ are depicted in Figs. 4 and 5. The density and the temperature profiles immediately reveal the inelastic nature of the collisions. In the elastic case $(e=1)$ both would simply be constant throughout the tube as mentioned above.

The qualitative features of the system are not too sensitive to the precise values of the control parameters $N$ and the inelasticity $\varepsilon$, provided that $N \geq 2, \varepsilon>0$, and $N \varepsilon<1$. Already for $N=2$, the steady state is found to consist of one commuting particle and one particle that remains close to the right wall [19]. The value of the third parameter $T_{0}$ (the driving strength) is not essential as it can be removed from the simulations by rescaling all particle velocities with $\sqrt{T_{0} / m}$. With respect to the inelasticity $\varepsilon$ one may anticipate that below some critical value $\varepsilon_{\text {crit }}$ (depending on $N$ ) the dissipation due to the collisions in the system will be overpowered and the cluster is fluidized [20]. For the values that we have used for $\varepsilon$ our system always shows a coexistence of 1 traveling particle and $N-1$ cluster particles as a steady state, although the transient toward this state can take a very long time.

At this point it is instructive to note that the individual behavior of the leftmost particle does not only cause the breakdown of hydrodynamics in this region (as was discussed in the introduction), but also triggers an interesting dynamical phenomenon [Fig. 3(a)]: once in a while this particle gets a particularly small velocity from the hot wall, giving the cluster time to expand; and after each expansion it takes a large number of collisions to force the cluster back to its ordinary size and density again. This intermittent expansion of the cluster has been treated in detail in Refs. $[11,19]$.

The extent of the expansion depends on the restitution coefficient $e$. For increasing $e$ the clustered particles show less spread in their velocities, causing the amplitude of the occasional expansions to decrease; see Fig. 3(b). Consequently, the boundary region between the diluted and dense regimes becomes narrower when $e \rightarrow 1$, yielding very sharp density and temperature profiles. This is illustrated in Figs. 4(a) and 4(b).

By contrast, the hydrodynamic model (discussed in detail in the next section) predicts that the boundary region be- 

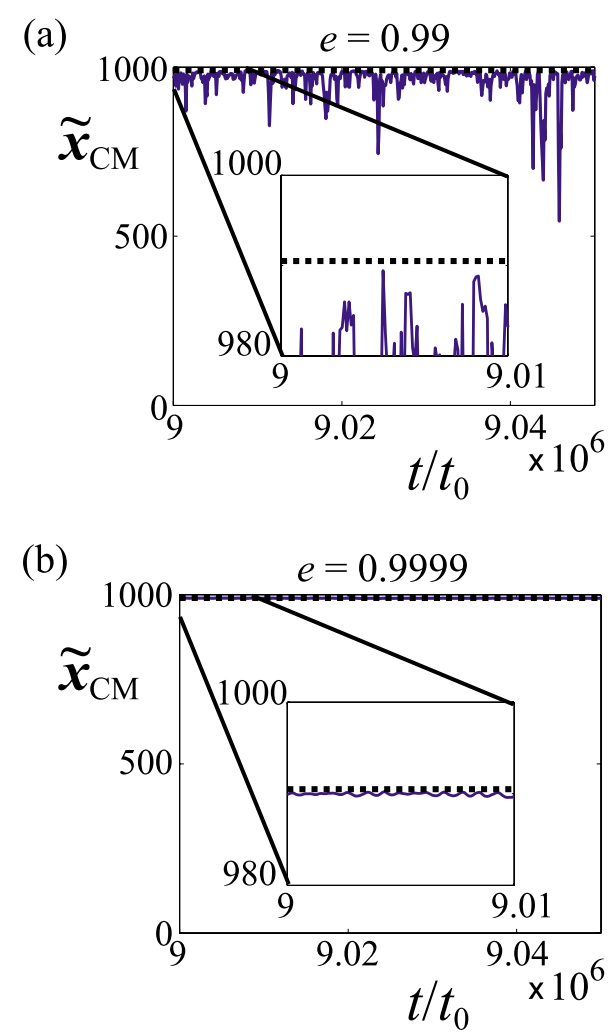

FIG. 3. (Color online) (a) Position of the cluster's center of mass, $\tilde{x}_{\mathrm{CM}}(t)=x_{\mathrm{CM}} / d$, as a function of time (normalized by $t_{0}=L \sqrt{m / T_{0}}$ ) for a MD simulation with $N=20$ particles and a restitution coefficient $e=0.99$. The cluster occasionally expands and recompacts; the expansions occur when the left particle picks up a particularly small velocity from the hot wall. The dotted line (see also inset) marks the maximal value of $x_{\mathrm{CM}}=L-d(N-1) / 2$ corresponding to a close-packed cluster in a tube of length $L=1000 d$. (b) For a higher restitution coefficient, $e=0.9999, x_{\mathrm{CM}}$ stays much closer to this maximal value.

comes wider for growing $e$ and thus gives increasingly smooth profiles in the approach toward the elastic limit $e=1$, as seen in Figs. 4(c) and 4(d). As far as the density profiles are concerned, this opposite limiting behavior is the only appreciable difference between the MD results and the hydrodynamic model; we will discuss it in depth in a forthcoming publication. The temperature profiles show a much more impressive difference: In the MD simulations the drop from $\tilde{T}=1$ to $\tilde{T}=0$ occurs in a relatively narrow interval, whereas the hydrodynamic model shows an almost linear decrease over the entire diluted interval. It is precisely this difference that will be discussed in the following sections.

\section{HYDRODYNAMICS OF THE STEADY STATE}

We consider the steady state of the system. This means that the full hydrodynamic problem [which would involve a number density $n(x, t)$, velocity field $u(x, t)$, and granular temperature $T(x, t)]$ here reduces to finding the two timeindependent quantities $n=n(x)$ and $T=T(x)$, while $u \equiv 0$. To achieve this, we use three hydrodynamics equations plus boundary conditions.

\section{MD simulations:}

(a)

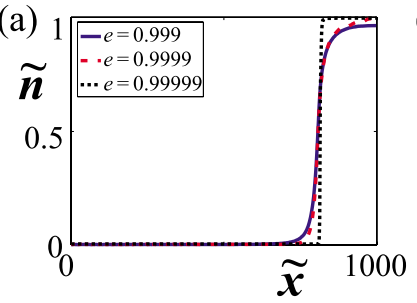

(b)

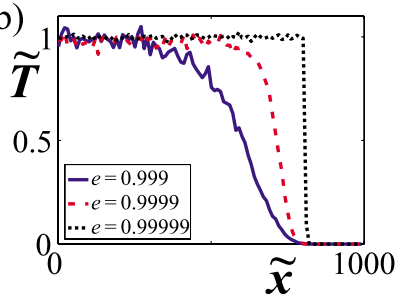

Hydrodynamics: (c)

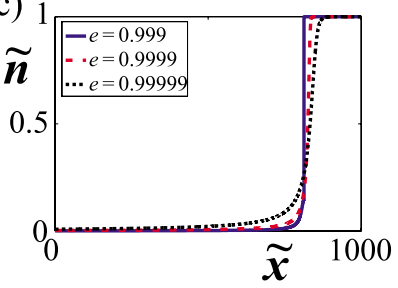

(d)

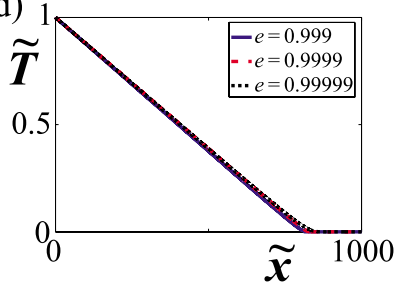

FIG. 4. (Color online) MD vs Hydrodynamics in the near-elastic limit for $N=187$ particles in a tube of length $L=1000 d$ : [(a) and (b)] Time-averaged dimensionless number density $\tilde{n}(\tilde{x})$ and granular temperature $\tilde{T}(\tilde{x})$ obtained from MD simulations for three different values of the restitution coefficient $e$. The total duration of each simulation was $t=5 \cdot 10^{7} t_{0}$ (but we discarded the transient behavior before the onset of the steady state), sampled every 100 time units. $[(\mathrm{c})$ and $(\mathrm{d})]$ Density $\widetilde{n}(\widetilde{x})$ and temperature $\widetilde{T}(\widetilde{x})$ from hydrodynamic theory.

We go beyond the ideal-gas description by incorporating the finite size of the particles (via the constitutive relations [21]) and also the dissipation due to the collisions. This is in the same spirit as we did for the granular Leidenfrost effect [10], an analogous clustering phenomenon in a twodimensional vertical system.

The first hydrodynamic equation is the momentum balance [22],

$$
\frac{d p}{d x}=0,
$$

where $p$ is the pressure. It immediately follows that $p$ is constant throughout the tube. Its value is determined by the second equation in our model, the equation of state,

$$
p=\frac{n T}{1-n d}=\frac{n T}{1-n / n_{c}} .
$$

Here $n_{c}$ is the maximal number density (i.e., the number of particles per unit length in the close-packed case, $n_{c}=1 / d$ ). In Eq. (4) one recognizes the ideal-gas law $p=N T / L=n T$ with a van der Waals correction for the excluded length due to the finite size of the particles, i.e., the free space within the tube is not $L$ but $L-N d$. It differs from the ideal-gas law used as the equation of state by $\mathrm{Du}$ et al. [11] since they used point particles.

The third hydrodynamic equation is the energy balance, expressing the steady state equilibrium between the heat flux through the array of particles and the dissipation due to the inelastic collisions, 


$$
-\frac{d \Phi(x)}{d x}=I(x) .
$$

Here $\Phi(x)=-\kappa(x) d T / d x$ is the heat flux (from high to low temperatures, hence the minus sign), with $\kappa(x)$ the thermal conductivity allowing for finite size effects,

$$
\kappa(x)=C_{1} \frac{T^{1 / 2}(x)}{n(x) \ell(x)}=C_{1} \frac{T^{1 / 2}(x)}{1-n(x) / n_{c}} .
$$

Here $C_{1}$ is a constant and $\ell(x)$ denotes the local mean free path, which is related to the number density as $n(x)=1 /[d$ $+\ell(x)]$, or equivalently $\ell(n(x))=\left[1-n(x) / n_{c}\right] / n(x)$. The dimensionless mean-free path $\ell(x) / d=\left[1-n(x) / n_{c}\right] /\left[n(x) / n_{c}\right]$, called the Knudsen number, is very large in the dilute region and vanishingly small within the cluster. Note that Du et al. used $\kappa(x) \propto T^{1 / 2}(x)$ for the thermal conductivity [11].

In Eq. (5) the dissipation rate $I$ (per unit length and per unit time) is given by

$$
I(x)=C_{2} \varepsilon \frac{n(x)}{\ell(x)} T^{3 / 2}(x)=C_{2} \varepsilon \frac{n^{2}(x) T^{3 / 2}(x)}{1-n(x) / n_{c}},
$$

with $C_{2}$ a constant. The expression for $I$ is equal to the energy loss in one collision $(\propto \varepsilon T)$ multiplied by the total number of collisions per unit time taking into account excluded volume $(\propto n \sqrt{T} / \ell)$ [23]. Du et al. used the low density limit of Eq. (7) for the dissipation rate, i.e., $I(x) \propto \varepsilon n^{2}(x) T^{3 / 2}(x)$ [11].

The set of three hydrodynamic Eqs. (3)-(5) is complemented by three boundary conditions: (i) the imposed granular temperature at the hot wall $T(0)=T_{0}$, (ii) vanishing heat flux at the insulating wall $\Phi(L)=0$, and (iii) conservation of particles $\int_{0}^{L} n(x) d x=N$.

Now let us introduce dimensionless variables,

$$
\tilde{n}=\frac{n}{n_{c}}, \quad \tilde{T}=\frac{T}{T_{0}}, \quad \tilde{x}=\frac{x}{d} .
$$

The force balance [Eq. (3)] and the equation of state [Eq. (4)], combined into one, then read

$$
\tilde{p}=\frac{\tilde{n} \tilde{T}}{1-\tilde{n}}=\text { constant }=\tilde{p}_{0},
$$

the energy balance [Eq. (5)] becomes (with $C=C_{2} / C_{1}$ )

$$
-\frac{d \tilde{\Phi}}{d \tilde{x}}=C \varepsilon \frac{\tilde{n}^{2} \widetilde{T}^{3 / 2}}{1-\tilde{n}}, \quad \text { where } \tilde{\Phi}=\frac{-\widetilde{T}^{1 / 2}}{(1-\tilde{n})} \frac{d \tilde{T}}{d \tilde{x}}
$$

and the dimensionless boundary conditions are

$$
\tilde{T}(0)=1, \quad \widetilde{\Phi}(L / d)=0, \quad \int_{0}^{L / d} \tilde{n} d \tilde{x}=N .
$$

One thus arrives at a problem consisting of two first-order differential equations [see Eq. (10)] and three unknown quantities $\tilde{n}, \tilde{T}$, and $\widetilde{\Phi}$. We use Eq. (9) to express $\tilde{n}$ in terms of $\tilde{T}[24]$,

$$
\tilde{n}(\widetilde{T})=\frac{1}{1-\widetilde{T}(\widetilde{x})[1-1 / \tilde{n}(0)]},
$$

and with this the two differential equations to be solved take the form

$$
\begin{aligned}
& \frac{d \tilde{T}}{d \tilde{x}}=-\frac{[1-\tilde{n}(\tilde{T})] \tilde{\Phi}}{\tilde{T}^{1 / 2}}, \\
& \frac{d \tilde{\Phi}}{d \tilde{x}}=-C \varepsilon \frac{\tilde{n}^{2}(\tilde{T}) \widetilde{T}^{3 / 2}}{1-\tilde{n}(\tilde{T})},
\end{aligned}
$$

supplemented by the boundary conditions $\tilde{T}(0)=1$ and $\widetilde{\Phi}(L / d)=0$. Using $\tilde{n}(0)$ [which is still contained in Eq. (12)] as a parameter, we solve the above boundary value problem numerically, varying the shooting parameter $\widetilde{\Phi}(0)$ to fulfill the second boundary condition. Once $\widetilde{T}(\widetilde{x})$ and $\widetilde{\Phi}(\widetilde{x})$ have been found, the number density $\tilde{n}(\tilde{x})$ is given by Eq. (12). Next, we turn to the third boundary condition of Eq. (11): integrating $\widetilde{n}(\widetilde{x})$ over the tube length yields the total number of particles $N$.

In the process of solving Eqs. (13) and (14) we find that, for all parameter values considered in the present study, the heat flux $\tilde{\Phi}(\tilde{x})$ vanishes already at some location before the right wall. Let us call this point $\tilde{x}=\tilde{x}_{1}$. The temperature $\tilde{T}(\tilde{x})$ becomes zero here, and [via Eq. (12)] the density $\tilde{n}(\tilde{x})$ becomes 1, giving a singularity in the Eqs. (13) and (14). As a result, the heat flux $\widetilde{\Phi}(\widetilde{x})$ gets negative beyond $\tilde{x}_{1}$, as if energy would flow from the cold right side into the system. For our time-averaged quantities this makes no physical sense. So beyond $\tilde{x}_{1}$ we fix the heat flux to $\widetilde{\Phi}(\widetilde{x})=0$, and hence also the values $\widetilde{T}(\widetilde{x})=0$ and $\tilde{n}(\tilde{x})=1$, which means that the interval between $\tilde{x}_{1}$ and the right wall is an immobile, close-packed cluster [25].

Apart from the heat flux itself, also its derivative $d \widetilde{\Phi} / d \widetilde{x}$ is zero at $\tilde{x}_{1}$, which means that the transition from the dilute region to the cluster is quite smooth.

The resulting density and temperature profiles are shown in Figs. 4 and 5. The agreement with the corresponding MD simulations [exploiting the only fit parameter in our theory, namely the constant $C$ in Eq. (14)] is seen to be good regarding the density $\tilde{n}$ : The hydrodynamical model correctly predicts the coexistence of a cluster and a highly diluted phase. However, the temperature $\widetilde{T}$ is wide of the mark in the dilute region. The model predicts a linear decrease of $\widetilde{T}$, whereas the actual temperature is constant [26]: this is a consequence of the fact that this region contains just one particle, i.e., a Knudsen gas, which has no way of exchanging energy with other particles until it meets the cluster at $\tilde{x}_{1}$. It has a constant velocity along its whole path. Therefore also the distribution of velocities cannot change in the Knudsen gas region, hence $\widetilde{T}$ is constant. 

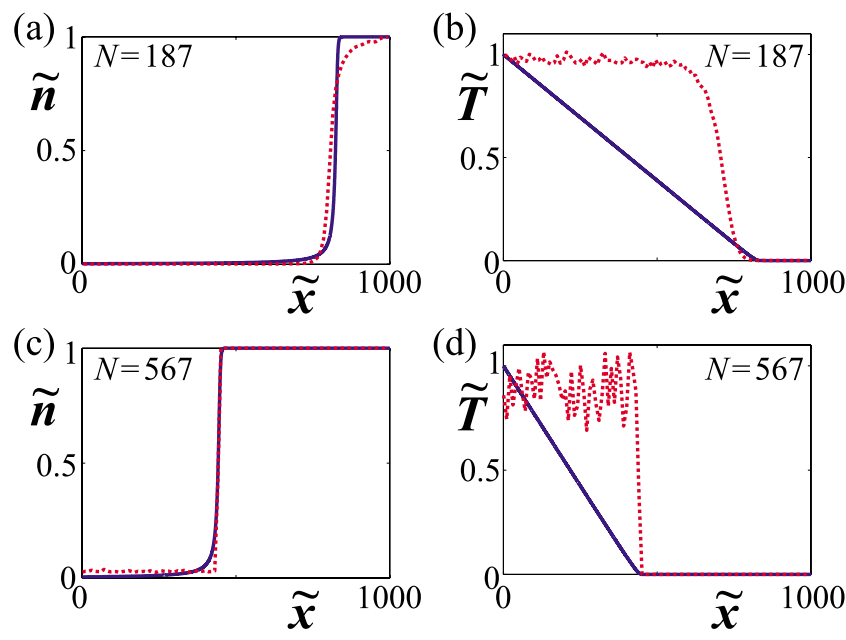

FIG. 5. (Color online) Density $\widetilde{n}(\widetilde{x})$ and temperature $\widetilde{T}(\widetilde{x})$ along a tube of length $L=1000 d$ for various numbers of particles: [(a) and (b)] $N=187$ and [(c) and (d)] $N=567$. The restitution coefficient is fixed at $e=0.9999$. Dashed red curves represent our MD simulations (total duration was $t / t_{0}=5 \times 10^{7}$ with sampling every 100 time units), and solid blue lines the hydrodynamic model (13) and (14).

The fact that the energy exchange takes place only at the boundary between the dilute region and the cluster is illustrated by Fig. 6, where we plot the thermal conductivity obtained from the hydrodynamic model,

$$
\widetilde{\kappa}(\widetilde{x})=\frac{\widetilde{T}^{1 / 2}(\widetilde{x})}{1-\widetilde{n}(\widetilde{T})}=\widetilde{T}^{1 / 2}(\widetilde{x})+\frac{\widetilde{n}(0)}{1-\widetilde{n}(0)} \widetilde{T}^{-1 / 2}(\widetilde{x})
$$

and the mean energy exchange between the particles, $E_{\text {exch }}(\widetilde{x})$. The latter quantity is determined from the MD simulation by keeping track of the energy gain or loss of the right particle for every colliding pair of particles within a region of size $d$ around the position $\tilde{x}$.

The thermal conductivity shows a pronounced upswing at the boundary $\widetilde{x}_{1}$. It is also nonzero to the left of this boundary $\left(\propto \widetilde{T}^{1 / 2}\right.$, i.e., the ideal-gas behavior for $\widetilde{n} \ll 1$ ), but this is an

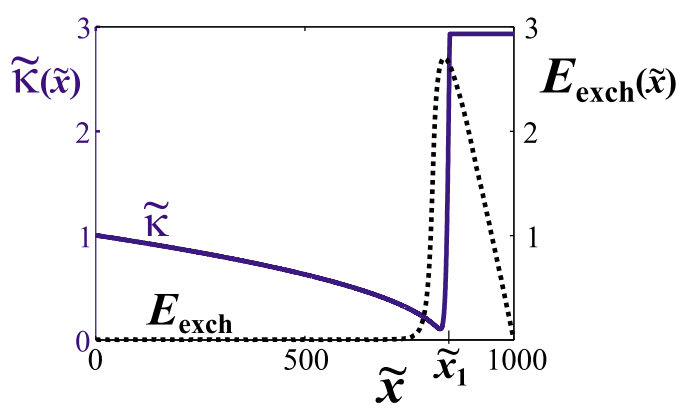

FIG. 6. (Color online) Energy transport through the system: hydrodynamics vs MD. The energy transport according to the hydrodynamic model is represented by the thermal conductivity $\widetilde{\kappa}(\widetilde{x})$ (solid blue line); it is calculated via Eq. (15) from the hydrodynamic temperature profile for $N=187$ particles of Figs. 5(a) and 5(b). The energy transport for the MD simulations is expressed by $E_{\text {exch }}(\widetilde{x})$ (dotted black line). artifact of the continuum description: the dilute region is treated as a hydrodynamic gas, which-even at low density-is by definition supposed to consist of an ensemble of particles with mutual energy exchange. The actual $\widetilde{\kappa}$ consists of the upswing only. That is, we are dealing with a Knudsen gas, i.e., a gas in which only the collisions with the boundaries contribute to the dynamics of the gas. This is confirmed by $E_{\text {exch }}(\widetilde{x})$ in the simulations, which in the dilute region shows no energy exchange at all, and a pronounced maximum at the boundary of the cluster $\widetilde{x}_{1}$. The density inside the cluster steadily grows, see Fig. 5(a), and as a result the number of collisions increases in the cluster. However, the energy involved in every collision drops drastically here and causes the energy exchange to decay linearly. At the right wall the energy exchange vanishes since the collisions of the rightmost particle with the wall are elastic. The hydrodynamic model does not predict the gradual growth of the density in the cluster, so $\widetilde{\kappa}(\widetilde{x})$ does not decrease but stays constant all the way up to the right wall.

\section{INCORPORATING THE LOCALIZED ENERGY EXCHANGE}

Treating the thermal conductivity $\widetilde{\kappa}(\widetilde{x})$ as a step function, the temperature drops from 1 , the boundary value $\widetilde{T}(0)$, to zero in one step at $\widetilde{x}_{1}$ :

$$
\widetilde{T}(\widetilde{x})= \begin{cases}1 & \text { for } 0<\tilde{x}<\tilde{x}_{1}, \\ 0 & \text { for } \tilde{x}_{1}<x<\frac{L}{d} .\end{cases}
$$

Here the value of $\tilde{x}_{1}$ is determined from the fact that the cluster contains $N-1$ immobile particles, closely packed against the right wall: $\tilde{x}_{1}=\frac{L}{d}-(N-1)$. The corresponding number density is

$$
\tilde{n}(\tilde{x})= \begin{cases}{\left[\frac{L}{d}-(N-1)\right]^{-1}} & \text { for } 0<\tilde{x}<\tilde{x}_{1}, \\ 1 & \text { for } \tilde{x}_{1}<x<\frac{L}{d} .\end{cases}
$$

It jumps from the small value $\left[\frac{L}{d}-(N-1)\right]^{-1}$, representing the commuting particle in the left part of the tube, at once to the close-packed value 1 .

In this hybrid model, in which hydrodynamics is blended with the individual behavior of the leftmost particle acting as a Knudsen gas, the force balance $d \widetilde{p} / d \widetilde{x}=0$ still holds throughout the system (so the pressure is constant). Also the equation of state Eq. (9) need not be altered (in the dilute part it determines the constant value of $\tilde{p}$, while in the solid part it gives an indeterminate result). The only adjustment has taken place in the energy balance [Eq. (10)]: the exchange of energy, which in the pure hydrodynamic model was supposed to occur along the whole length of the tube, has now been localized to $\tilde{x}_{1}$.

The density and temperature according to this modified model are compared with the MD results in Fig. 7. Not only the density profiles match well, as in the case of the purely hydrodynamic model, but also the temperature $\widetilde{T}(\widetilde{x})$ shows 

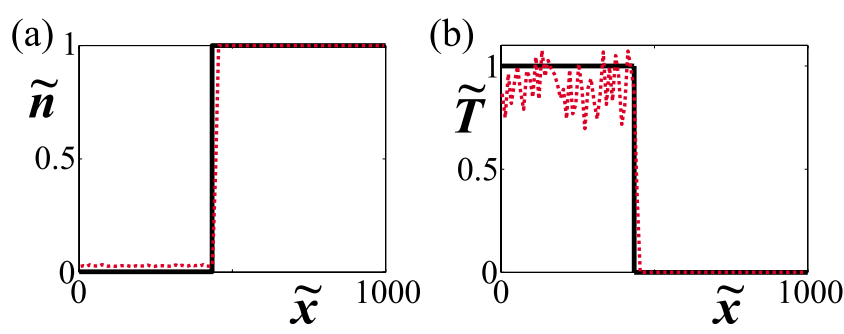

FIG. 7. (Color online) Same MD simulation data as in Figs. 5(c) and 5(d) (i.e., for $N=567$ particles, dashed red lines) but here the hydrodynamic curves are calculated with the localized-energyexchange model of Eqs. (16) and (17) (solid black lines). Both the density $\widetilde{n}(\widetilde{x})$ and the temperature $\widetilde{T}(\widetilde{x})$ now show good agreement.

good agreement. At this point it is worthwhile to note that the temperature in the MD simulations in Fig. 7(b) is slightly less than 1. This is because, whereas the average kinetic energy of the leftmost particle is equal to $T_{0}$ while it is moving to the right, it must be slightly lower when the particle returns from the cluster: it regains its momentum only after a series of (dissipative) collisions in the cluster [27].

The abruptness of the transition is a typical onedimensional feature: only in one dimension a single particle is capable of controlling a cluster, whereas in higher dimensions particles can pass each other leading to qualitatively different behavior [1]. If the current system is extended to more than one dimension, the transition from the dilute region to the cluster becomes less sharp. The dilute region then consists of more than one particle, allowing (next to the collisions with the boundaries) also internal collisions between the particles; so the pure Knudsen gas of the onedimensional case attains a more hydrodynamiclike character in higher dimensions. Experiments in a two-dimensional system of spherical particles rolling on a smooth surface and driven by a moving side wall indeed show a softer boundary between the diluted region and the cluster [28], and timedependent hydrodynamic theory was shown to give a reasonably accurate description of the dynamic behavior [29,30]. Related two-dimensional granular gases were quantitatively described in hydrodynamic terms in Refs. [10,31]. In three dimensions the hydrodynamic description may be anticipated to gain even more ground.

\section{CONCLUSION}

So we have answered the question to what extent hydrodynamics works in the granular system of $\mathrm{Du}, \mathrm{Li}$, and Kadanoff [11]: it successfully captures the density, with its sharp division in a dilute and a clustered region, but not the temperature. This can be traced back to the basic assumption of the continuum approach that the dilute region-even when the density gets very low-is supposed to consist of a sufficiently large number of particles to justify its treatment as a continuous medium. In the present system this assumption is incorrect since the dilute region contains only one particle, i.e., a Knudsen gas. It is at the level of the energy exchange that the discrepancy really makes a difference: where the continuum view would have an energy exchange throughout the dilute region (and a corresponding linear decrease in the granular temperature), the energy of the commuting particle remains in fact constant until it meets the cluster.

We introduced a localized-energy-exchange model which keeps the strong points of the hydrodynamic description (the force balance as well as the equation of state incorporating excluded volume effects) mixed with the Knudsen gas feature that all energy exchange takes place at the cluster boundary. This model gives an accurate description of both the density and the temperature.

Thus, we have employed the horizontal array of inelastic particles to explore the limits of granular hydrodynamics. Du et al. [11] introduced it as a system for which hydrodynamics simply breaks down, but there is more to it than that: it identifies the exact point at which the continuum description starts to fail and, on top of this, the reason why and how this shortcoming can be overcome.

\section{ACKNOWLEDGMENTS}

This work is part of the research program of FOM, which is financially supported by NWO.
[1] L. P. Kadanoff, Rev. Mod. Phys. 71, 435 (1999).

[2] J. T. Jenkins and M. W. Richman, Phys. Fluids 28, 3485 (1985).

[3] S. Luding, Phys. Rev. E 63, 042201 (2001).

[4] I. Goldhirsch and G. Zanetti, Phys. Rev. Lett. 70, 1619 (1993).

[5] J. Eggers, Phys. Rev. Lett. 83, 5322 (1999).

[6] J. J. Brey, F. Moreno, R. García-Rojo, and M. J. RuizMontero, Phys. Rev. E 65, 011305 (2001).

[7] E. Khain and B. Meerson, Phys. Rev. E 67, 021306 (2003).

[8] Y. Forterre and O. Pouliquen, J. Fluid Mech. 467, 361 (2002).

[9] D. Lohse, R. Bergmann, R. Mikkelsen, C. Zeilstra, D. van der Meer, M. Versluis, K. van der Weele, M. van der Hoef, and H. Kuipers, Phys. Rev. Lett. 93, 198003 (2004).

[10] P. Eshuis, K. van der Weele, D. van der Meer, and D. Lohse,
Phys. Rev. Lett. 95, 258001 (2005).

[11] Y. Du, H. Li, and L. P. Kadanoff, Phys. Rev. Lett. 74, 1268 (1995).

[12] E. L. Grossman and B. Roman, Phys. Fluids 8, 3218 (1996).

[13] J. J. Brey, M. J. Ruiz-Montero, and F. Moreno, Phys. Rev. E 62, 5339 (2000).

[14] B. Meerson, T. Pöschel, and Y. Bromberg, Phys. Rev. Lett. 91, 024301 (2003).

[15] An interesting variation is to consider particles of unequal mass, see J. J. Wylie and Q. Zhang, C. R. Math. 339, 603 (2004); Phys. Rev. E 74, 011305 (2006).

[16] From the point of view of the simulation technique, the fact that the particles have a finite diameter $d$ is immaterial since a one-dimensional system of size $L$ containing $N$ particles of 
diameter $d$ can be uniquely mapped onto a system of length $L-N d$ containing $N$ sizeless particles. The finite size of the particles does become important however as soon as one extracts hydrodynamic variables from the simulation results: in order to determine the particle density correctly one has to take the finite size of the particles into account.

[17] S. McNamara and W. R. Young, Phys. Fluids A 4, 496 (1992).

[18] Such a linearly corrected Gaussian distribution has been identified as the appropriate stochastic forcing at the wall for this type of systems by K. Geisshirt, P. Padilla, E. Præstgaard, and S. Toxvaerd, Phys. Rev. E 57, 1929 (1998).

[19] J. Yang, Phys. Rev. E 61, 2920 (2000).

[20] J. M. Pasini and P. Cordero, Phys. Rev. E 63, 041302 (2001).

[21] C. Vamoş, N. Suciu, and A. Georgescu, Phys. Rev. E 55, 6277 (1997).

[22] For the granular Leidenfrost effect [10], this balance had the form $d p / d x=-m g n$, but since we now deal with a horizontal system the gravity term is absent.

[23] E. L. Grossman, T. Zhou, and E. Ben-Naim, Phys. Rev. E 55, 4200 (1997).

[24] From Eq. (9) one finds $\tilde{n}=\widetilde{p_{0}} /\left(\widetilde{p_{0}}+\widetilde{T}\right)$, and with $\widetilde{p}_{0}=\widetilde{n}(0) /[1$ $-\tilde{n}(0)][$ since $\widetilde{T}(0)=1]$ this yields Eq. (12).

[25] This is not only a proper description of the steady state profiles but also (for the value $e=0.9999$ used here) a good approximation of the full time-dependent system; cf. Fig. 3(b)
[26] Since the pressure $p \approx n(x) T(x)$ in the dilute region is constant in both MD simulation and theory we also find small differences in the density profile (hardly discernible in Fig. 5), namely, that in the model the density slowly increases toward the cluster whereas the density in the hydrodynamic model remains approximately constant.

[27] In fact, the momentum is regained by the leftmost particle after $2(N-1)$ collisions in the cluster (remembering that the collision with the right wall is elastic). In each of these collisions the momentum transfer to the next particle is equal to $[1-(1-e) / 2]$, in leading order in the small parameter $(1-e)$. If the incoming velocity is equal to $v_{1}$, the magnitude of the regained velocity is given by $v_{1}^{\prime}=[1-(1-e) / 2]^{2(N-1)} v_{1} \approx[1-(N-1)(1-e)] v_{1}$. The average temperature in the dilute region thus approximately is equal to $[1-(N-1)(1-e)] T_{0}$. For the parameter values used in Fig. 7 $(N=567$ and $e=0.9999)$ this amounts to an energy loss of approximately $5 \%$.

[28] A. Kudrolli, M. Wolpert, and J. P. Gollub, Phys. Rev. Lett. 78, 1383 (1997).

[29] E. Livne, B. Meerson, and P. V. Sasorov, Phys. Rev. E 66, 050301(R) (2002).

[30] E. Livne, B. Meerson, and P. V. Sasorov, Phys. Rev. E 65, 021302 (2002).

[31] J. J. Brey and D. Cubero, Phys. Rev. E 57, 2019 (1998). 